\title{
EXPECTATIVAS DEL USUARIO DE SERVICIOS DE MOTOTAXI PARA LA PROMOCIÓN DEL TURISMO RECEPTIVO EN LA CIUDAD DE TARAPOTO
}

\section{EXPECTATIONS OF THE USER OF MOTOTAXI SERVICES FOR THE PROMOTION OF RECEPTIVE TOURISM IN THE CITY OF TARAPOTO}

Recepción: 2019-12-21 - Aceptación: 2020-05-25

\section{Jose Joel Cruz Tarrillo1; a}

(iD) ORCID iD 0000-0002-6372-5055

José Tarrillo Paredes ${ }^{1 ;}$ b

(iD) ORCID iD 0000-0003-3229-3189

\section{Carmelino Almestar Villegas ${ }^{1 ; c}$}

(iD) ORCID iD 0000-0002-1757-8832

1 Universidad Peruana Unión.

a Maestro en Administración de Negocios con Mención en Gestión Empresarial.

b Maestro en Investigación y Docencia Universitaria.

c Ingeniero Agroindustrial.

\section{RESUMEN}

Objetivo: Conocer las expectativas del turista como usuario del servicio de mototaxi en la ciudad de Tarapoto, mediante la técnica de análisis de componentes principales. Método: Se desarrolló una investigación de enfoque cuantitativo, de nivel descriptivo, con diseño de investigación no experimental de corte transversal. La muestra estuvo conformada por 150 turistas usuarios del servicio de mototaxi. La variable "expectativas del servicio de mototaxi", fue agrupada en cuatro componentes principales que recogen el 59,03\% de la varianza total explicada. Resultados: Los componentes principales encontrados para el modelo se denominan: componente 1, cumplimiento de normas del conductor; componente 2, comodidad del pasajero; componente 3, imagen personal del conductor y la componente 4, respeto al medio ambiente. Conclusiones: El turista usuario del servicio de mototaxi espera prioritariamente el cumplimiento de normas del conductor y la comodidad del viaje, ya que estos dos componentes explican el 39\% de la variable.

Palabras clave: Expectativas; Normas del conductor; Comodidad; Imagen personal.

\section{ABSTRACT}

Objective: To know the expectations of the tourist as a user of the motorcycle taxi service in the city of Tarapoto, using the principal component analysis technique. Method: A descriptive level research was analyzed, with a non-experimental cross-sectional research design. The sample was made up of 150 tourists using the motorcycle taxi service. The variable "motorcycle taxi service expectations" was grouped into four main components that include $59.03 \%$ of the total variance explained. Results: The main components found for the model are called: component 1, driver compliance; component 2, passenger comfort; component 3, personal image of the driver and component 4, respect for the environment. Conclusions: The tourist user of the motorcycle taxi service expects priority compliance with the rules of the driver and the comfort of the trip, since these two components explain $39 \%$ of the variable.

Keywords: Expectations; Driver's standards; Comfort; Personal image. 


\section{INTRODUCCIÓN}

Estudiosos de marketing han argumentado que las empresas deben cumplir o superar las expectativas del cliente con el fin de lograr la satisfacción del mismo. A menudo, las expectativas del turista pueden ser injustificadas, inviables, o improductivas de satisfacer. Si bien es cierto que, éstas deben ser adaptadas, en lugar de cumplidas, para ello es necesario conocer las expectativas del turista, como usuario del servicio de mototaxi, para que de esta manera poder diseñar un servicio acorde con sus necesidades.

De acuerdo con la Organización Mundial del Turismo (2020), el turismo es una herramienta que permite unir a grupos humanos de diferentes latitudes geográficas, generando con ello riqueza y cuidado del ambiente.

Jingjing y Nijkamp (2018), enfatizan que el turismo receptivo puede ser una fuerza impulsora nueva y poderosa para la innovación regional, mientras que el efecto del turismo receptivo en la innovación tecnológica parece ser más débil que el de la innovación social.

Por otro lado, el turismo receptivo en el Perú, es generador de divisas, ya que, en el 2019, generó US \$5300 millones. Asimismo, la Región San Martín en el año 2018 registró el mayor número de turistas nacionales y extranjeros, con un total de 452352 arribos, representando el 33\% del número de llegadas a la macro-región del oriente. Asimismo, la comisión de promoción del Perú para la Exportación y el Turismo (PROMPERÚ), es un organismo que tiene por finalidad, promover el turismo receptivo, fomentando la imagen del Perú en temas turísticos y de exportación (El Peruano, 2019).

En el año 2018, los lugares visitados de la provincia de San Martín por turistas extranjeros representan el 37\% del total de turistas que visitaron la región San Martín. Los visitantes provienen principalmente de Estados Unidos, Francia y España (Ministerio de Comercio Exterior y Turismo, 2019).

Actualmente, las empresas de servicios dan mayor énfasis en la satisfacción de las necesidades de los clientes. En sector transporte en la ciudad de Tarapoto este ámbito económico esta descuidada por lo que no se están cumpliendo adecuadamente las expectativas de los usuarios, al solucionar este problema, se tendría una mayor demanda de pasajeros de este servicio.

El parque automotor de la ciudad de Tarapoto el mototaxi tiene una participación del $75 \%$, por lo que hace meritorio investigar en este tema. En concordancia con Parasuranman, los atributos que causan mayor impresión en el cliente no son los relacionados al servicio en sí; sino los que conllevan el proceso de la prestación del mismo (Morais, Santos, Rizatto y Melleiro, 2013).

Se han realizado algunas investigaciones en Colombia sobre el servicio y reglamentación de transporte de carga y pasajeros en motocarro (castillo, 2010). Pero aún no se conoce investigaciones que aborden el tema de las expectativas de los usuarios de servicio de mototaxi.

Para Urdaneta y Joheni (2012), la política nacional de transporte urbano contribuye a mejorar el acceso al servicio de transporte público a los sectores de escasos recursos económicos, contribuyendo de esta manera a la inclusión social, esto permitirá reducir el precio de transporte urbano en la ciudad de Tarapoto.

La hipótesis que se plantean en el presente trabajo de investigación, es, que el cliente de servicio de mototaxi, siempre tiende a importarle más la seguridad que la prontitud en llegar al destino (Saldarriaga, 2011). Además, Kotler (2010) menciona que la clave para retener a los clientes es la satisfacción de sus expectativas. Un cliente muy satisfecho se mantiene leal más tiempo, compra más, habla favorablemente acerca de la empresa y sus productos, presta menos atención a la competencia y es menos sensible al precio.

De acuerdo con Coye (2004), los consumidores de servicios tienen expectativas acerca de lo que van a recibir del sistema de entrega del servicio. Estas expectativas son creencias acerca del servicio que percibirán, y se presume que influirán en la satisfacción y la evaluación de la calidad del servicio en general, por esta razón conocer las expectativas que tiene el usuario de servicios de mototaxi, es esencial, a la hora de prestar el servicio.

Asimismo, Peralta (2006), enfatiza el rol que juegan las expectativas, particularmente las expectativas afectivas, en la formación del juicio de satisfacción de los consumidores o usuarios de un servicio y en la percepción de la calidad del servicio, esto está relacionado con el trato amable que debe tener el conductor de mototaxi con el pasajero. 
Esta investigación tiene como objetivo conocer las expectativas del turista, usuario del servicio de mototaxi en la ciudad de Tarapoto. Esto permitirá diseñar un servicio acorde a los intereses del cliente y de esta manera mejorar la demanda de turistas.

\section{MATERIAL Y MÉTODOS}

\section{Tipo de investigación}

Se desarrolló una investigación de enfoque cuantitativo, de nivel descriptivo, con diseño de investigación no experimental de corte transversal. La población objetivo está conformada por los turistas usuarios del servicio de mototaxi de la ciudad de Tarapoto, particularmente turistas que arribaron al destino turístico Laguna Azul (Ministerio de Comercio Exterior y Turismo, 2019).

\section{Población}

Las personas encuestadas, fueron turistas nacionales y extranjeros usuarios del servicio de mototaxi con edades comprendidas entre 18 a 50 años, que arribaron al destino turístico Laguna Azul. De acuerdo con el Ministerio de Comercio Exterior y Turismo (2019), la población de turistas que arribaron al destino turístico antes mencionado fueron 290 turistas en el mes de junio. Así las unidades de estudio, estuvieron conformadas por hombres y mujeres, de todos niveles educativos y socioeconómicos.

\section{Muestra}

El tamaño de muestra se calculó de acuerdo con Aguilar (2005), para estudios de naturaleza descriptiva, con variable categórica, se utiliza la siguiente ecuación:

$$
\begin{gathered}
\mathrm{n}=\frac{\mathrm{z}_{0}{ }^{2 * p} \mathrm{p} * \mathrm{q} * \mathrm{~N}}{\mathrm{e}^{2 *}(\mathrm{~N}-1)+\mathrm{z}_{0}{ }^{2 *} \mathrm{p} * \mathrm{q}} \\
\mathrm{n}=\frac{3.8416 * 0.5 * 0.5 * 250}{0.0025 *(250-1)+3.8416 * 0.5 * 0.5} \\
\mathrm{n}=165
\end{gathered}
$$

Siendo:

$\mathrm{n}$ : Tamaño de la muestra

$\mathrm{N}$ : Tamaño de la población

$Z_{0}$ : Valor de distribución normal estándar con probabilidad (1- $\left.\alpha / 2\right)$

p: Probabilidad de éxito (sugerencia: tomar $p=q=0.5$ )

q: Probabilidad de fracaso

e: Error de muestreo

Al reemplazar los datos en la ecuación, se obtuvo un tamaño de muestra de 165 turistas. Sin embargo, quince de ellos eran turistas que aún no habían alcanzado la mayoría de edad, por tanto, no fueron encuestados, quedando de esta manera una muestra de 150 turistas. De los cuales $70(46,7 \%)$ fueron nacionales y $80(53,3 \%)$ fueron extranjeros, el levantamiento de los datos se realizó en hoteles y restaurantes de la ciudad de Tarapoto, considerando que el turista ha hecho uso del servicio de mototaxi previamente. Asimismo, del total de turistas, 66 (44\%) fueron hombres y 84 (56\%) fueron mujeres. Se utilizó un muestreo aleatorio simple, siendo la población 245 turistas que arriban a la ciudad de Tarapoto (Ministerio de Comercio Exterior y Turismo, 2019).

\section{Instrumentos}

Se construyó un cuestionario basado en el método de completar las frases, que es una derivación del instrumento clásico de Likert, en la cual se incluyen frases incompletas respecto a la variable de estudio y se incluye una puntuación que va del 0 al 10; donde el encuestado mide la fuerza del atributo (Hernández, Fernández y Baptista, 
Tabla 1

Percepción de las expectativas del servicio de mototaxi

\begin{tabular}{|c|c|c|c|}
\hline Componentes & Nivel & Frecuencia & Porcentaje \\
\hline \multirow{4}{*}{ Amabilidad } & Deficiente & 1 & $1 \%$ \\
\hline & Aceptable & 4 & $3 \%$ \\
\hline & Bueno & 145 & $97 \%$ \\
\hline & Total & 150 & $100 \%$ \\
\hline \multirow{4}{*}{ Limpieza de asientos } & Deficiente & 1 & $1 \%$ \\
\hline & Aceptable & 4 & $3 \%$ \\
\hline & Bueno & 145 & $97 \%$ \\
\hline & Total & 150 & $100 \%$ \\
\hline \multirow{4}{*}{ Higiene del conductor } & Deficiente & 2 & $1 \%$ \\
\hline & Aceptable & 3 & $2 \%$ \\
\hline & Bueno & 145 & $97 \%$ \\
\hline & Total & 150 & $100 \%$ \\
\hline \multirow{4}{*}{ Equipo de sonido } & Deficiente & 90 & $60 \%$ \\
\hline & Aceptable & 48 & $32 \%$ \\
\hline & Bueno & 42 & $28 \%$ \\
\hline & Total & 150 & $100 \%$ \\
\hline \multirow{4}{*}{ Precios cómodos } & Deficiente & 5 & $3 \%$ \\
\hline & Aceptable & 13 & $9 \%$ \\
\hline & Bueno & 132 & $88 \%$ \\
\hline & Total & 150 & $100 \%$ \\
\hline \multirow{4}{*}{ Facilidad para otorgar vueltos } & Deficiente & 5 & $3 \%$ \\
\hline & Aceptable & 7 & $5 \%$ \\
\hline & Bueno & 138 & $92 \%$ \\
\hline & Total & 150 & $100 \%$ \\
\hline \multirow{4}{*}{ Documentación } & Deficiente & 1 & $1 \%$ \\
\hline & Aceptable & 2 & $1 \%$ \\
\hline & Bueno & 147 & $98 \%$ \\
\hline & Total & 150 & $100 \%$ \\
\hline \multirow{4}{*}{ Respeto por las reglas de tránsito } & Deficiente & 1 & $1 \%$ \\
\hline & Aceptable & 1 & $1 \%$ \\
\hline & Bueno & 148 & $99 \%$ \\
\hline & Total & 150 & $100 \%$ \\
\hline \multirow{3}{*}{ Respeto por el ambiente } & Aceptable & 1 & $1 \%$ \\
\hline & Bueno & 149 & $99 \%$ \\
\hline & Total & 150 & $100 \%$ \\
\hline \multirow{4}{*}{ Prontitud } & Deficiente & 12 & $8 \%$ \\
\hline & Aceptable & 48 & $32 \%$ \\
\hline & Bueno & 90 & $60 \%$ \\
\hline & Total & 150 & $100 \%$ \\
\hline \multirow{4}{*}{ Revistas para leer } & Deficiente & 48 & $32 \%$ \\
\hline & Aceptable & 34 & $23 \%$ \\
\hline & Bueno & 68 & $45 \%$ \\
\hline & Total & 150 & $100 \%$ \\
\hline
\end{tabular}


2014). Dicho instrumento está conformado por doce ítems, cuya validez y confiabilidad fue obtenida mediante el coeficiente alfa de Cronbach, el cual fue aceptable de 0,60 (Ibíd., 2005). El cuestionario se diseñó teniendo en cuenta las dimensiones de la calidad del servicio de transporte urbano, indicadas por Barboza y Torres (2017), estas dimensiones fueron: atención al cliente, tiempo de viaje, trato al usuario, forma de manejo, capacitación al conductor, confort, impacto ambiental, estado físico del vehículo, servicio ofertado, información, costo de viaje, paradas, accesibilidad y comodidad. Los autores mencionados utilizaron un cuestionario de 18 preguntas para la recolección de los datos. Asimismo, menciona que el cuestionario fue utilizado para medir la calidad del servicio de transporte urbano en México y Nepal.

\section{Análisis de datos}

Para analizar los datos se utilizó técnicas estadísticas descriptivas como frecuencias y porcentajes, las cuales se presentaron en tablas de frecuencia. Asimismo, se utilizó, la técnica del análisis de componentes principales (ACP), para determinar las expectativas del servicio de mototaxi que esperan recibir los usuarios. El Análisis de Componentes Principales, es un método que busca la reducción de las variables y eliminación de superposiciones de un conjunto de datos (Vasconcelos, 2010). Asimismo, Santo (2012) la técnica de ACP permite identificar patrones en los datos y expresarlos de una manera tal que sus semejanzas y diferencias, sean destacadas. Para analizar los datos, se utilizó el paquete estadístico SPSS 22. De acuerdo con Bernal, Martínez y Sánchez (2003) el gráfico de sedimentación permite conocer el número de componentes a retener. Este criterio establece que deben retenerse las componentes, situadas antes de la zona de sedimentación. Esta zona queda fijada a partir de la componente que no presentan pendientes elevadas en el gráfico de Autovalor versus componente. Esto comportamiento se hace notorio a partir de la componente 4 o 5 .

\section{RESULTADOS}

A continuación, se presenta los principales hallazgos de la investigación. En la tabla 1, se presenta los resultados descriptivos de los componentes estudiados, en dónde los turistas perciben que la amabilidad, limpieza de los asientos e higiene, precios cómodos, la facilidad para otorgar vueltos, la documentación, el respeto por las reglas de tránsito, respeto por el ambiente, prontitud, son calificados como bueno; no así sucede con el equipo de sonido en donde su calificación es deficiente y con respecto a revistas para leer, existe un $32 \%$ que califica como deficiente; un $23 \%$ como aceptable y un $45 \%$ como bueno.

Asimismo, en la tabla 2, se observa que la variable, expectativas del servicio de mototaxi, está conformado por cuatro componentes que recogen el $59,03 \%$ de la varianza explicada y son los que tienen autovalores mayores que 1. Hay dos componentes que explican el $39 \%$ del modelo expectativas del servicio de mototaxi,el primer componente explica que el usuario tiene como prioridad que el conductor conozca y cumpla con las normas de seguridad establecidas y el segundo componente es que el usuario espera tener comodidad al momento de hacer uso del servicio.

Tabla 2

Varianza total explicada del constructo expectativas del servicio de mototaxi.

\begin{tabular}{lllll}
\hline$N^{\circ}$ & Componente & Auto valor total & $\%$ de varianza & \% acumulado \\
\hline 1 & Normas del conductor & 2,65 & 22,04 & 22,04 \\
2 & Comodidad del pasajero & 2,04 & 17,01 & 39,05 \\
3 & Imagen personal del conductor & 1,34 & 11,13 & 50,18 \\
4 & Respeto al medio ambiente & 1,06 & 8,85 & 59,03 \\
\hline
\end{tabular}

Para tener una apreciación más adecuada en la figura 1, se presenta que a partir del componente 4, respeto al medio ambiente, la pendiente de la línea tiende a ser constante, por esta razón se considera como componentes principales que explican la variable expectativa del servicio de mototaxi, son 4, como se muestra en la tabla 2.

Igualmente, en la figura 2, se observa que la variable expectativa del servicio de mototaxi, puede agruparse en cuatro componentes principales, a saber: El componente cumplimiento de normas del conductor, el turista necesita seguridad al momento de hacer uso del servicio, cortesía por parte del conductor, el vehiculo tiene que estar completamente limpio, los documentos tanto de la movilidad, conductor tienen que estar en regla, el 
conductor tiene que conocer y respectaer las normas de transito. Por otro lado, el segundo componente refleja que los turistas desean que el vehiculo cuente con musica para disfrutar durante el viaje, que los precios sean cómodos, cuente con sencillo al momento de dar vuelto que y que el mototaxi tenga material de lectura como revistas entre otros. Asimismo, el tercer componente el turista sugiere que el conductor mantega su imagen personal. Finalmente, el cuarto componente, el turista desea que el conductor respete el medio ambiente y prontitud en el viaje. Estos resultados están de acuerdo con Parasuranman, quien sugiere que los atributo que causan mayor impresión en el cliente no son los relacionados al servicio en sí, sino los que conllevan el proceso de la prestación del mismo (Morais, Santos, Rizatto y Melleiro, 2013).

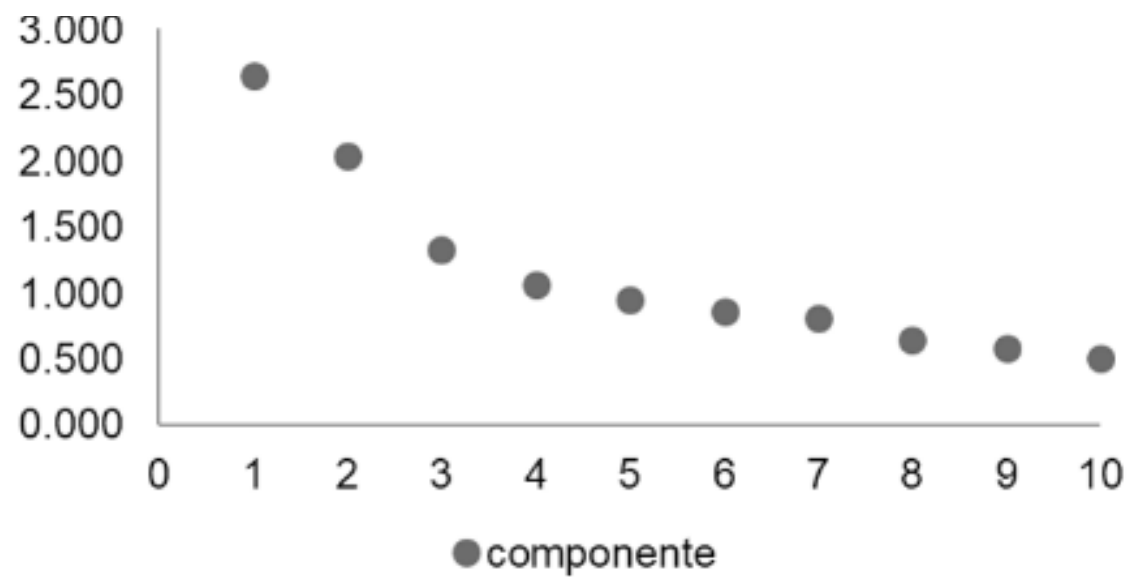

Figura 1. Gráfico de sedimentación de la variable expectativas del servicio de mototaxi.

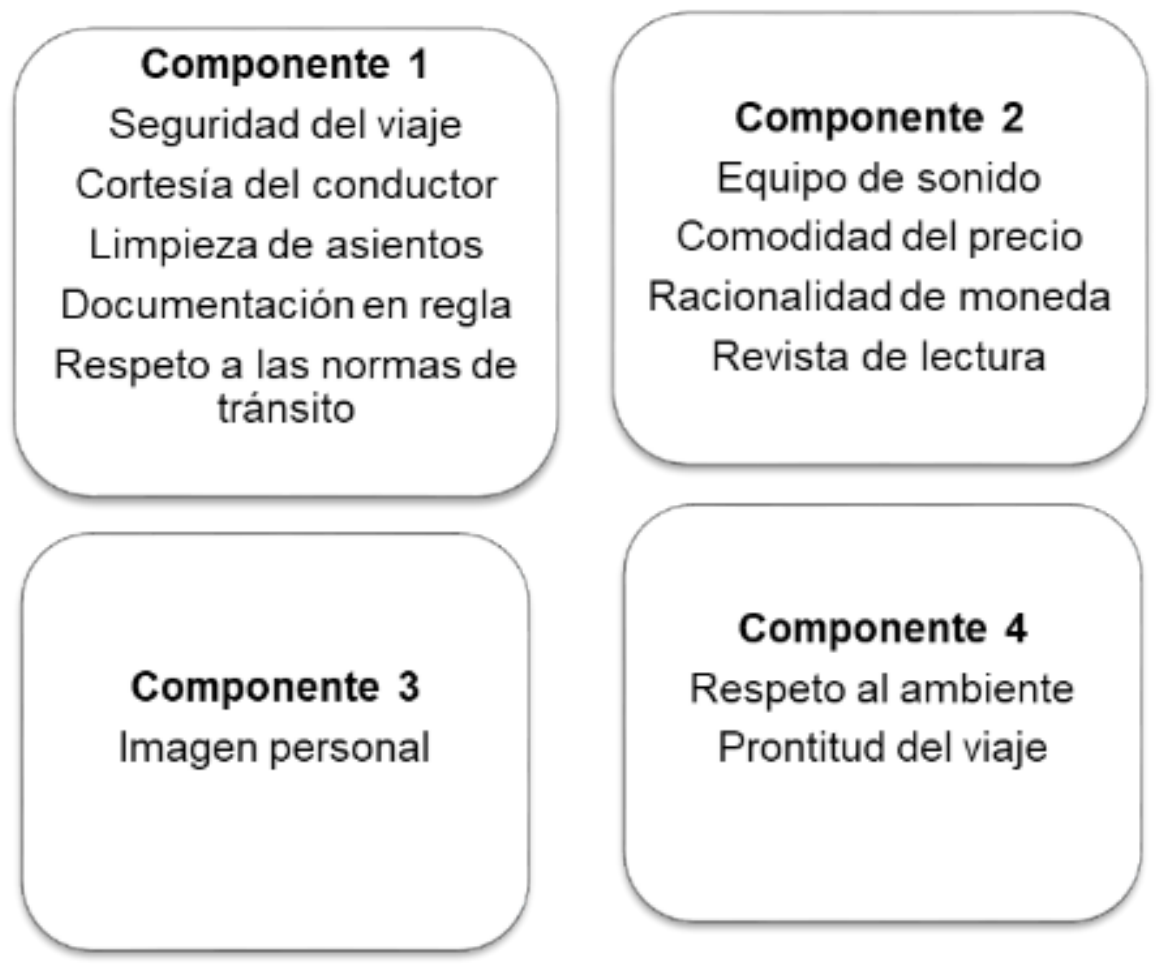

Figura 2. Componentes principales de la variable expectativas del servicio de mototaxi. 


\section{DISCUSIÓN}

De acuerdo con el propósito de investigación fue conocer las expectativas del turista usuario del servicio de mototaxi en la ciudad de Tarapoto, según lo expuesto en los resultados, se observa que el componente cumplimiento de normas del conductor, explica el $22,04 \%$ de la variable expectativas del servicio de mototaxi. Esta dimensión agrupa los siguientes sub-factores: Seguridad del viaje, cortesía del conductor, limpieza de asientos, documentación en regla y respeto a las normas de tránsito. Acorde con este resultado, Barboza y Torres (2017) indican que el cumplimiento de regulaciones y el buen trato por parte de los conductores, son factores que afectan la calidad del servicio de transporte urbano, percibido por los usuarios.

Los mismos autores indican que si no se cumple con atributos como la higiene de la unidad móvil, protección del ambiente, estado físico de las paradas, falta de cortesía para mujeres embarazadas y falta de información, el resultado será una pérdida de la calidad del servicio de trasporte de pasajeros. Por esta razón, empresas como la Asociación de "Moto Taxis Manco Capac" de la ciudad de Tarapoto, han implementado charlas de educación ambiental para sus asociados, cumplimiento de regulaciones y mejora de la imagen personal y del vehículo.

Al cumplir las expectativas del usuario de servicio de mototaxi, satisfará dos dimensiones claves de la calidad del servicio, enunciadas por Gronroos, a saber, el aspecto técnico, que significa qué servicio está siendo proporcionado y cómo el servicio es entregado (Jingjing y Nijkamp, 2018). Sin embargo, Guglielmetti, Toni, Raharjo, Di Pietro y Petros (2018), afirman que la satisfacción del cliente presenta tres variables: valor funcional, valor emocional y valor social. Asimismo, estos autores indican que la percepción de la calidad del servicio afecta significativamente la satisfacción del consumidor.

Por esta razón, Peralta (2006), enfatiza el rol que juegan las expectativas afectivas, en la formación del juicio de satisfacción de los usuarios de un servicio y en la percepción de la calidad del servicio, lo cual está relacionado con el trato amable que debe tener el conductor de mototaxi, con el pasajero. La cuestión ambiental, también es un tema de interés por los usuarios de servicio de mototaxi, en la ciudad de Tarapoto.

De acuerdo con Guglielmetti, Toni, Raharjo, Di Pietro, y Petros (2018), la calidad del servicio y la actitud ambiental afectan la intención de los individuos hacia la movilidad sostenible. De otro lado Eka (2016) muestra que la percepción pública de los servicios de transporte público con indicadores de seguro, empatía, confiabilidad, capacidad de respuesta, material y comodidad afecta la calidad del servicio. Santos (2010), destaca como atributos del trasporte urbano colectivo el tiempo total del viaje y la comodidad dentro del vehículo.

Para Khurshid (2012), existe una relación positiva entre la calidad del servicio y la satisfacción del cliente, en el trasporte público de pasajeros. Asimismo, Barbosa y Silva (2017), indican como atributos que valora el usuario de trasporte urbano a los siguientes: estado de las vías; características de los lugares de parada; la comunicación; almacenamiento; tiempo de viaje; coste del paso; accesibilidad a portadores de necesidades especiales; la seguridad; la puntualidad del servicio. Por otro lado, de acuerdo con Saldarriaga (2011), el cliente de servicio de motocarro, siempre tiende a importarle más la seguridad que la prontitud en llegar al destino.

Asimismo, la variable expectativa del servicio de mototaxi, fue agrupada en cuatro componentes principales que recogen el 59,03\% de la varianza total explicada. Los componentes principales encontrados para el modelo se denominan: Componente 1, cumplimiento de normas del conductor; componente 2, comodidad del pasajero; componente 3 , imagen personal del conductor y componente 4, respeto al medio ambiente. Finalmente, el turista usuario del servicio de mototaxi espera prioritariamente el cumplimiento de normas del conductor y la comodidad del viaje, ya que estos dos componentes explican el $39 \%$ de la variable. esta información será de mucha utilidad para aquellos que se dedican a prestar este servicio ya que podrán satisfacer las necesidades del usuario de manera adecuada. Asimismo, los resultados de esta investigación abren las puertas a otros estudios más específicos, como el diseño del servicio de mototaxi en la ciudad de Tarapoto.

\section{REFERENCIAS BIBLIOGRÁFICAS}

Barbosa, J., \& Da Silva, S. (2018). Critérios de qualidade em serviços de transporte público urbano: uma contribuição teórica. Recuperado el 10 de octubre de 2018 de http://periodicos.ufes.br/bjpe/article/view/v4n1_6/ pdf

Castillo, B. (2010). El mototaxismo ¿propblema informal o u medio de generación de ingresos para resolver en parte el problema del desempleo en montería. Revista económicas, 31(31). Recuperado de: https://revistascientificas.cuc.edu.co/economicascuc/article/view/1185/921 
Coye, R. (2004). Managing customer expectations in the service encountered. International Journal of Service Industry Management, 15(1), 54-71. Recuperado el 17 de mayo de 2018 de: https://doi. org/10.1108/09564230410523330

El Peruano. (23 de febrero de 2019). Resolución ministerial, Nº55-2019-MINCETUR, poder ejecutivo, Comercio Exterior y Turismo - Autorizan viaje de representante de PROMPERÚ a Colombia, en comisión de servicios-resolución ministerial-N 055-2019-MINCETUR. Recuperado el 28 de enero de 2020 de: https://vlex.com. $\mathrm{pe} / \mathrm{vid} / 768388849$

Eka, K. (2016). The Effect of Public Transport Services on Quality of Life in Medan City. Recuperado el 10 de octubre de 2018 de: https://reader.elsevier.com/reader/sd/pii/S1877042816315099?token=4C84AA1AC3CD21C5890DBF72309248D28F0306D09ABDE2A14843BE6820423E0408E87777FCCD7830FB40319A03F33592

Hernández, R., Fernández, C., \& Baptista, P. (2014). Metodología de investigación científica (5a edición). México DF: McGraw Hill. Recuperado de: http://www.esup.edu.pe/descargas/dep_investigacion/Metodologia\%20 de\%20la\%20investigaci\%C3\%B3n\%205ta\%20Edici\%C3\%B3n.pdf

Khurshid, R. (2012). Service quality and customer satisfaction in public transport sector of Pakistan: An Empirical Study. International Journal of Economics and Management Sciences. Recuperado de: https://www. omicsonline.org/open-access/service-quality-and-customer-satisfaction-in-public-transport-sector-of-pakistan-an-empirical-study-.php?aid=17202

Kotler, P. (2010). Fundamentos del marketing (6a de edición). México DF, México: Prentice Hall.

Jingjing, L., \& Nijkamp, P. (2018). Inbound Tourism as a Driving Force for Regional Innovation: A Spatial Impact Study on China. A Spatial Impact Study on China. Journal of Travel Research, 1(1), 1-14. Recuperado de: https://doi.org/10.1177/0047287518771223

Ministerio de Comercio Exterior y Turismo. (2019). Reporte regional de turismo san martín. Recuperado de: https://www.gob.pe/institucion/mincetur/informes-publicaciones/365503-reportes-de-turismo-reporte-regional-de-turismo-san-martin-2019

Morais, M., Santos, D., Rizatto, D., \& Melleiro, M. (2013). The use of the quality model of Parasuraman, Zeithaml and Berry in health services. Jurnal of School of Nursing. Recuperado el 10 de octubre de $2018 \mathrm{de:} \mathrm{http://www.}$ scielo.br/pdf/reeusp/v47n5/0080-6234-reeusp-47-05-1227.pdf

Guglielmetti, R., Toni, M., Raharjo, H., Di Pietro, L., \& Petros, S (2018). Does the service quality of urban public transport enhance sustainable mobility?. Journal of Cleaner Production. Recuperado de: https://doi. org/10.1016/j.jclepro.2017.11.052

Organización Mundial del Turismo. (22 de enero de 2020). El rey de españa muestra un gran apoyo a las ambiciones de la OMT. Recuperado de: https://www.unwto.org/es/el-rey-de-espana-muestra-un-gran-apoyo-a-lasambiciones-de-la-omt

Peralta, J. (2006). Rol de las expectativas en el juicio de satisfacción y calidad percibida del servicio. Límite,1(14), 195-214. https://www.redalyc.org/pdf/836/Resumenes/Resumen_83601409_1.pdf

Saldarriaga, A. (2011). Modelación de la elección del motocarro como medio de transporte público en zonas rurales aplicación al municipio de Girardota. (Tesis de maestría). Facultad de Minas, Universidad Nacional de Colombia. Recuperado de: http://bdigital.unal.edu.co/5466/1/71213617.2011.pdf

Santo, R. (2012). Utilização da Análise de Componentes Principais na compressão de imagens digitais. Einstein,10(2), 135-139. Recuperado de: http://www.scielo.br/pdf/eins/v10n2/pt_a04v10n2.pdf

Dos Santos, P. (2010). A percepção de importância dos atributos do transporte coletivo. Recuperado el 10 de octubre de 2018. Recuperado de: https://www.lume.ufrgs.br/bitstream/handle/10183/28584/000769209.pdf?sequence $=1$ 
Supo, J. (2016). Cómo validar un instrumento. La guía para validar un instrumento en 10 pasos. Recuperado de: http://www.cua.uam.mx/pdfs/coplavi/s_p/doc_ng/validacion-de-instrumentos-de-medicion.pdf

Urdaneta, G., \& Joheni, A. (2012). El transporte público urbano en Venezuela: ¿hacia la inclusión social? Revista de Ciencias Sociales, 18(3), 449-461. Recuperado de https://www.redalyc.org/pdf/280/28024392005.pdf

Vasconcelos, S. (2010). Análise de componentes principais. Recuperado el 10 de octubre de: http://www2.ic.uff. $\mathrm{br} /$ aconci/PCA-ACP.pdf

Correo elctronico: jose.cruz@upeu.edu.pe 